\title{
Effects of SARS-CoV-2 infection on neuroimaging and neurobehavior in neonates
}

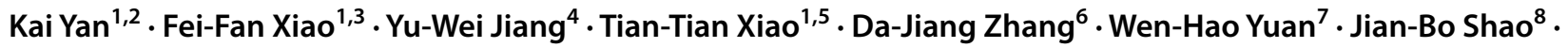 \\ Guo-Qiang Cheng ${ }^{1,2} \cdot$ Ling-Kong Zeng $^{7}$
}

Received: 26 October 2020 / Accepted: 23 February 2021 / Published online: 19 March 2021

(c) Children's Hospital, Zhejiang University School of Medicine 2021

\begin{abstract}
Background We collected neonatal neurological, clinical, and imaging data to study the neurological manifestations and imaging characteristics of neonates with coronavirus disease 2019 (COVID-19).

Methods This case-control study included newborns diagnosed with COVID-19 in Wuhan, China from January 2020 to July 2020. All included newborns had complete neurological evaluations and head magnetic resonance imaging. We normalized the extracted T2-weighted imaging data to a standard neonate template space, and segmented them into gray matter, white matter, and cerebrospinal fluid. The comparison of gray matter volume was conducted between the two groups.

Results A total of five neonates with COVID-19 were included in this study. The median reflex scores were 2 points lower in the infected group than in the control group $(P=0.0094)$, and the median orientation and behavior scores were 2.5 points lower in the infected group than in the control group $(P=0.0008)$. There were also significant differences between the two groups in the total scale score $(P=0.0426)$. The caudate nucleus, parahippocampal gyrus, and thalamus had the strongest correlations with the Hammersmith neonatal neurologic examination (HNNE) score, and the absolute correlation coefficients between the gray matter volumes and each part of the HNNE score were all almost greater than 0.5 .

Conclusions We first compared the neurological performance of neonates with and without COVID-19 by quantitative neuroimaging and neurological examination methods. Considering the limited numbers of patients, more studies focusing on the structural or functional aspects of the virus in the central nervous system in different age groups will be carried out in the future.
\end{abstract}

Keywords Coronavirus disease $2019 \cdot$ Magnetic resonance imaging · Neonates · Quantitative neuroimaging · Severe acute respiratory syndrome coronavirus 2

Ling-Kong Zeng

freeman315@163.com

1 Department of Neonatology, Children's Hospital of Fudan University, National Children's Medical Center, Shanghai, China

2 Key Laboratory of Neonatal Diseases of Ministry of Health, Shanghai, China

3 Center for Molecular Medicine, Children's Hospital of Fudan University, National Children's Medical Center; Institutes of Biomedical Sciences, Fudan University, Shanghai, China

4 Institute of Neuroscience, Key Laboratory of Primate Neurobiology, CAS Center for Excellence in Brain Science and Intelligence Technology, Chinese Academy of Sciences, Shanghai, China
5 Department of Neonatology, Chengdu Women's and Children's Central Hospital, School of Medicine, University of Electronic Science and Technology of China, Chengdu, China

6 Department of Radiology, Children's Hospital of Fudan University, National Children's Medical Center, Shanghai, China

7 Department of Neonatology, Wuhan Children's Hospital (Wuhan Maternal and Child Healthcare Hospital), Tongji Medical College, Huazhong University of Science and Technology, Wuhan, China

8 Department of Radiology, Wuhan Children's Hospital (Wuhan Maternal and Child Healthcare Hospital), Tongji Medical College, Huazhong University of Science and Technology, Wuhan, China 


\section{Introduction}

Coronavirus disease 2019 (COVID-19), caused by severe acute respiratory syndrome coronavirus 2 (SARS-CoV-2), has spread around the world, and its superior transmission has taken humanity's public health system by surprise. Since the first case of SARS-CoV-2 infection was reported, SARS-CoV-2 is known to primarily attack the human lung. Subsequent clinical findings [1,2] suggest that SARS-CoV-2 may attack the central nervous system (CNS) in humans.

In fact, medical professionals have identified several patients with non-specific neurological symptoms that manifest as headaches [3] from the beginning of the epidemic in January 2020. A subset of patients with COVID19 developed symptoms that included central and peripheral nervous system symptoms $[2,4,5]$. Studies have found evidence of SARS-CoV-2 in patient's cerebrospinal fluid (CSF) [6] and in neuronal cells and vascular endothelial cells from autopsies [7]. A recent study further showed the presence of SARS-CoV-2 RNA in the brains of infected patients, and the virus can directly affect nerve cells in the brainstem and cerebellum [8]. Surprisingly, Vivanti et al. [9] provided a comprehensive case report demonstrating that SARS-CoV-2 is trans-placental, that the infected neonate had neurological symptoms along with bilateral periventricular white matter (WM) and subcortical glial hyperplasia as well as cerebral vasculitis, and that the long-term neurological prognosis of the child remains unknown, although later follow-up revealed a reduction in brain damage. In a recent systematic review of SARS-CoV-2-infected neonates, the authors reported that $18 \%(18 / 176)$ of infected neonates had neurological symptoms [10].

Previously, we reported several cases of neonates with COVID-19, and we summarized the epidemiological and clinical characteristics of the infected neonatal population $[11,12]$. Because we are now concerned about the potential neurological effects of SARS-CoV-2, we collected neonatal neurological, clinical, and imaging data to further study the neurological manifestations and imaging characteristics of neonates with COVID-19.

\section{Methods}

\section{Study design and participants}

This study was a case-control study, and included newborns diagnosed with COVID-19 in Wuhan, China from January to July 2020. All included newborns had complete neurological evaluations and head magnetic resonance imaging (MRI). The study excluded cases in which family members did not agree to undergo MRI testing. We matched 15 normal neonatal head MRIs at a ratio of $1: 3$ according to gestational age as normal controls (excluding those with neurological and respiratory diseases), and we compared the brain volumes of different brain regions between the case group and the control group. This study was approved by the hospital ethics committee and complied with the Declaration of Helsinki.

\section{Data collection}

We collected perinatal and epidemiological information of newborns, including maternal prenatal characteristics (epidemiological characteristics, fever, medication, gestational hypertension, gestational diabetes, amniotic fluid, fetal membrane, placenta, umbilical cord, etc.). The demographic and clinical treatment information of newborns comes from the health information system.

\section{Neonatal neurological examination}

Five neonates diagnosed with COVID-19 underwent standard neonatal neurologic examination with reference to the Hammersmith neonatal neurologic examination (HNNE), including cranial nerve function score, posture score, movement score, tone score, reflexes, and reactions. We recorded the measurements on a table, scoring them on a 5-point scale to obtain raw scores.

\section{Laboratory test}

The laboratory test indicators including a routine blood panel, blood gas analysis, electrolytes, liver and kidney function, myocardial enzyme spectrum, procalcitonin, interleukin-6, blood culture, respiratory etiology, TORCH screen (toxoplasmosis, rubella cytomegalovirus, herpes simplex, human immunodeficiency virus), throat swab, and anal swab. During the hospitalization period, all laboratory examinations were performed by the central hospital laboratory.

\section{Virus test}

The mothers of these newborns had been diagnosed with COVID-19 pneumonia prenatally. At the time of admission of the newborns, we collected samples of their throat swabs and anal swabs to be sent to the Chinese Center for Disease for nucleic acid testing. SARS-CoV-2 infection status was tested by real-time reverse transcription polymerase chain reaction (novel coronavirus PCR fluorescence diagnostic kit, BGI, China) in the Chinese Center for Disease Control and Prevention. Procedures for the 
collection, transfer, and processing of the samples met the requirements of the World Health Organization. SARS$\mathrm{CoV}-2$ testing for neonates was performed at birth and every 3 days until two consecutive results were negative for SARS-CoV-2.

\section{Lung imaging exam}

Newborns underwent a chest X-ray examination within 24 hours after admission. Newborns with thickened lung texture or pulmonary exudation underwent lung computed tomography as well. The imaging doctor completed the reading and the report.

\section{Neuroimaging exam}

The neonate's cranial MR was refined within one week after vital signs were stable (off oxygen). Brain MRI was performed with a Siemens Magnetom Espree 1.5-T scanner (Siemens Healthineers, Erlangen, Germany) using phased array surface coils. The scanning sequence includes T1-weighted, T2-weighted, and diffusion-weighted imaging. Infants were first fed, tightly swaddled, and had earplugs inserted; and then infants were scanned during sleep. Before the scan, the vital signs of the neonates were stable. To prevent artifacts caused by head movement, all newborns received phenobarbital at a dose of $5 \mathrm{~mL} / \mathrm{kg}$ with parental consent and kept quiet. Infants were monitored using an oxygen saturation probe. The images were analyzed by a pediatric neurologist and by radiologists at the Children's Hospital of Fudan University.

\section{Ages and stages questionnaires assessment}

Developmental outcomes were assessed after the corrected ages of 9 months using the ages and stages questionnaires, third edition (ASQ-3). The scale is divided into five domains: communication, gross motor, fine motor, problem solving, and personal social. Each domain has six items, and each item has three answers: yes, sometimes yes, and no, for a score of 10,5 , and 0 , respectively. The sum of the scores of the six items for each region is the score for that region, and the sum of the scores of the five regions is the total score for this scale. One or more scores in the grid's dark-shaded zone indicate that the child may need further assessment. Scores in the light-shaded "monitoring" zone help identify children at risk. Scores outside the shaded zones indicate that the child is doing well in these areas. More than two domains $<2$ standard deviations below the mean area were considered developmental delay [13]. Considering the risk of hospital-acquired infections by bringing the newborn to the hospital for follow-up, we completed this assessment by telephone follow-up.

\section{Definition of diagnosis}

COVID-19 cases were confirmed based on the 7th edition of the new coronavirus pneumonia prevention and control protocol for COVID-19 released by the national health commission of the People's Republic of China. The discharge criteria were based on the neonatal expert consensus and the 7 th edition of the new coronavirus pneumonia prevention and control protocol for COVID-19.

\section{Data processing}

All T2-weighted imaging (T2WI) data were checked visually for apparent artifacts, motion blurs and outliers, and the data with such problems were excluded. For the 20 usable T2WI datasets ( 15 controls and 5 patients), we extracted the brain tissues from the whole head using the FMRIB Software Library (FSL 6, http://www.fmrib.ox.ac.uk/fsl). Voxelbased morphometry (VBM) analyses of the extracted T2WI data were performed using Statistical Parametric Mapping (SPM12, http://www.fil.ion.ucl.ac.uk/spm). We first normalized the extracted T2WI data to a standard template space for neonates, and we segmented them into gray matter (GM), WM, and CSF. We then smoothed the GM volumes images using a Gaussian filter with a kernel of full width at half maximum of $5 \mathrm{~mm}$. Finally, we performed a two-sample $t$ test on the smoothed GM volumes images between the control and patient groups. The cutoff threshold value for statistical significance was set at $P<0.001$ (uncorrected) and a cluster size $>50$ voxels.

\section{Statistical analysis}

We used STATA 15.0 (StataCorp, College Station, TX, USA) to complete the statistical analysis. Measurement data are expressed as means and standard deviations, and a Wilcoxon signed-rank test was used to compare measurement data between the two groups. Count data are expressed by frequency, and the Chi-squared test was used to compare count data between the two groups. $P<0.001$ (uncorrected) was statistically significant. In image analysis, we performed a two-sample $t$ test on the smoothed GM volumes images between the control and patient groups. The cutoff threshold value for statistical significance was set at $P<0.001$ (uncorrected) and a cluster size $>50$ voxels. The Spearman correlation tests and scatter plots were used to examine the correlation between regional GM volumes and HNNE scores. Before conducing a logistic regression analysis, we did a collinearity analysis of the data for the 12 brain regions volumes. We analyzed the collinearity and calculated the eigenvalues and eigenvectors of the correlation matrix using the kappa function and the eigen function by $R$ language ( $R$ version 4.0.3). A kappa value $>100$ indicates that the model 
has severe collinearity. We calculated the kappa value of the model as 11.0053 . Thus, by the kappa value we found that the collinearity of the model is small. We then used the eigen function to obtain the eigen value of the matrix. The minimum of the eigenvalues was 0.07563118 . Because the coefficients of the equations did not differ significantly, while the results of the equations tended to zero, no collinearity was found between the volumes of the individual brain regions.

\section{Results}

\section{Chain of temporal evidence of neurological symptoms in the general COVID-19 population}

We reviewed the available literature, and several studies have reported that patients with COVID-19 may exhibit neurological symptoms, including CNS symptoms (headache, dizziness, impaired consciousness, ataxia, acute cerebrovascular disease, and epilepsy) and peripheral nervous system symptoms (loss of taste, loss of smell, loss of vision, and neuropathic pain). Of these neurological symptoms, "loss of smell" is the most common. At the same time, scientists have identified SARS-CoV-2 in CSF, neuronal cells, and cerebral vascular endothelial cells. A case of WM damage was reported in a neonate with COVID-19. The specific temporal chain of evidence is shown in Supplementary Fig. 1.

\section{Baseline for confirmation of COVID-19 in newborns}

This article included a total of five newborns infected by SARS-CoV-2, including two males and three females. The median gestational age was 39 weeks (range 31-40 weeks). The results of demographic and laboratory examinations of the five neonates with COVID-19 are shown in Table 1. In the infected group, the proportion of elevated procalcitonin and the proportion of characteristic changes in chest fluoroscopy were all higher than that in the control group $(P<0.05)$.

\section{Evaluation of neurological behavior}

On neonatal neurological examination, the median reflex scores were 2 points lower in the infected group than that in the control group $(P=0.0094)$, and the median orientation and behavior scores were 2.5 points lower in the infected group than in the control group $(P=0.0008)$. There were also significant differences between the two groups in total scale scores $(P=0.0426)$. No statistically significant differences were seen between the two groups for the remainder of the comparison $(P>0.05$, Table 2$)$. However, the 9-monthold ASQ screening results did not show signs of neurodevelopmental delay, and although neonate 4 had the lowest scores, the scores were still in the normal range (Table 3 ). These neonates require more prolonged neurodevelopmental follow-up.

\section{Neuroimaging features}

Among the five neonates with COVID-19, four neonates presented with WM changes, including two full-term neonates (neonate 1 and neonate 3 ) who had hypoxic changes. As shown in Supplementary Fig. 2, there are signal changes of varying intensity in the basal ganglia region in T1WI. One neonate (neonate 4) and one full-term neonate (neonate 5) had brain hypoplasia with delayed myelination. MRI of the other neonates with COVID-19 revealed no significant abnormalities.

\section{Comparison of voxel morphology in different brain regions}

We compared the T2WI of the two groups by VBM (GM volumes) and found that there was a decrease in brain volume in the para hippocampal gyrus (PHG), orbitofrontal cortex (ORB), anterior cingulate gyrus (ACG), superior frontal gyrus (SFG), caudate nucleus of the basal junction region $(\mathrm{CN})$, and thalamic region in both groups (Supplementary Fig. 3). The inferior frontal gyrus (IFG), middle frontal gyrus (MFG), Rolandic operculum (ROL), middle temporal gyrus (MTG), and precuneus showed different degrees of brain volume increase. Both the thalamus and the precuneus showed bilateral changes in volume.

\section{Correlation analysis of differential brain region volumes with neurobehavioral scores}

We correlated the brain regions with significant differences in GM volumes with HNNE scores. The distribution of HNNE scores among the 20 neonates are showed in Supplementary Fig. 4a, and the correlation coefficients between the variables are presented in the heat map in Supplementary Fig. 4b. The results showed that the CN, PHG, and thalamus had the strongest correlation with the HNNE score, and the absolute correlation coefficients between the GM volumes and each part of the HNNE score were almost all greater than 0.5 . The correlations between the HNNE reflex score and the brain volume of the $\mathrm{CN}$, PHG, and thalamus were $0.61,0.64$ and 0.75 , respectively; the correlations between the HNNE orientation and behavior scores with the $\mathrm{CN}$, PHG, and thalamic brain volume were $0.57,0.64$, and 0.69 , respectively. The correlations of HNNE total score with $\mathrm{CN}$, PHG, and thalamic brain volume were $0.62,0.70$, and 0.70 , respectively (Supplementary Fig. 4c). 
Table 1 Baseline information and clinical and lung radiologic features of neonates exposed to SARS-CoV-2

\begin{tabular}{|c|c|c|c|c|}
\hline Characteristics & Total & Neonates with COVID-19 & Control & $P$ \\
\hline \multicolumn{5}{|l|}{ Neonatal information } \\
\hline Age of admission to NICU (h), median (range) & $60(8-432)$ & $72(18-432)$ & $54(8-144)$ & $>0.05$ \\
\hline Gestational age (wk), median (range) & $38(31-41)$ & $39(31-40)$ & $38(35-41)$ & $>0.05$ \\
\hline Male/female & $11 / 9$ & $2 / 3$ & $9 / 6$ & $>0.05$ \\
\hline Birth weight $(\mathrm{kg})$, median (range) & $3.25(2.87-4.12)$ & $3.35(3.03-3.36)$ & $3.21(2.87-4.12)$ & $>0.05$ \\
\hline Twins, $n(\%)$ & $1(5)$ & $1(20)$ & $0(0)$ & $>0.05$ \\
\hline SGA, $n(\%)$ & $0(0)$ & $0(0)$ & $0(0)$ & $>0.05$ \\
\hline Asphyxia, $n(\%)$ & $1(5)$ & $1(20)$ & $0(0)$ & $>0.05$ \\
\hline \multicolumn{5}{|l|}{ Clinical course } \\
\hline Symptomatic cases, $n(\%)$ & $3(15)$ & $3(60)$ & $0(0)$ & $>0.05$ \\
\hline Fever, $n(\%)$ & $2(10)$ & $2(40)$ & $0(0)$ & $>0.05$ \\
\hline Lethargy, $n(\%)$ & $2(10)$ & $2(40)$ & $0(0)$ & $>0.05$ \\
\hline Any respiratory symptoms, $n(\%)$ & $1(5)$ & $1(20)$ & $0(0)$ & $>0.05$ \\
\hline Any gastrointestinal symptoms, $n(\%)$ & $1(5)$ & $1(20)$ & $3(20)$ & $>0.05$ \\
\hline PCIS < $90, n(\%)$ & $1(5)$ & $1(20)$ & $0(0)$ & $>0.05$ \\
\hline Oxygen support, $n(\%)$ & $1(5)$ & $1(20)$ & $0(0)$ & $>0.05$ \\
\hline Ventilatory support, $n(\%)$ & $1(5)$ & $1(20)$ & $0(0)$ & $>0.05$ \\
\hline Antibiotics, $n(\%)$ & $1(5)$ & $1(20)$ & $3(20)$ & $>0.05$ \\
\hline Antiviral drugs, $n(\%)$ & $0(0)$ & $0(0)$ & $0(0)$ & $>0.05$ \\
\hline Days to achieve discharge criteria (d), median (range) & $16(5-28)$ & $27(12-28)$ & $11(5-21)$ & $>0.05$ \\
\hline Early-onset COVID-19, $n(\%)$ & $4(20)$ & $4(80)$ & $0(0)$ & - \\
\hline Mortality, $n(\%)$ & $0(0)$ & $0(0)$ & $0(0)$ & $>0.05$ \\
\hline \multicolumn{5}{|l|}{ Chest X-ray, $n(\%)$} \\
\hline Opacity or opacities, or densities & $3(15)$ & $3(60)$ & $0(0)$ & 0.009 \\
\hline Infiltrate & $3(15)$ & $3(60)$ & $0(0)$ & 0.009 \\
\hline Pneumonia & $5(25)$ & $5(100)$ & $0(0)$ & $<0.001$ \\
\hline Consolidation & $0(0)$ & $0(0)$ & $0(0)$ & $>0.05$ \\
\hline Ground-glass & $1(5)$ & $1(20)$ & $0(0)$ & $>0.05$ \\
\hline Pneumothorax & $0(0)$ & $0(0)$ & $0(0)$ & $>0.05$ \\
\hline Effusion & $0(0)$ & $0(0)$ & $0(0)$ & $>0.05$ \\
\hline Transient tachypnea of newborn & $0(0)$ & $0(0)$ & $0(0)$ & $>0.05$ \\
\hline \multicolumn{5}{|l|}{ Maternal information } \\
\hline Lab-confirmed cases, $n(\%)$ & $5(25)$ & $5(100)$ & $0(0)$ & $>0.05$ \\
\hline Clinical diagnosed cases, $n(\%)$ & $0(0)$ & $0(0)$ & $0(0)$ & $>0.05$ \\
\hline Age (y), median (range) & $28(25-33)$ & $28(25-33)$ & $28(26-31)$ & $>0.05$ \\
\hline Confirmed COVID-19 at third trimester, $n(\%)$ & $5(25)$ & $5(100)$ & $0(0)$ & $>0.05$ \\
\hline Delivery mode (CS), $n(\%)$ & $10(50)$ & $3(60)$ & $7(47)$ & $>0.05$ \\
\hline Meconium stained AF, $n(\%)$ & $4(20)$ & $2(40)$ & $2(13)$ & $>0.05$ \\
\hline Premature rupture of membranes, $n(\%)$ & $0(0)$ & $0(0)$ & $0(0)$ & $>0.05$ \\
\hline Chorioamnionitis, $n(\%)$ & $0(0)$ & $0(0)$ & $0(0)$ & $>0.05$ \\
\hline Co-infected pathogens, $n(\%)$ & $0(0)$ & $0(0)$ & $0(0)$ & $>0.05$ \\
\hline \multicolumn{5}{|l|}{ Initial laboratory results, $n(\%)$} \\
\hline White blood cells $<5$ or $>30\left(\times 10^{9} / \mathrm{L}\right)$ & $1(5)$ & $1(20)$ & $0(0)$ & $>0.05$ \\
\hline Lymphocytes $<2\left(\times 10^{9} / \mathrm{L}\right)$ & $1(5)$ & $1(20)$ & $0(0)$ & $>0.05$ \\
\hline Platelets $<150\left(\times 10^{12} / \mathrm{L}\right)$ & $1(5)$ & $1(20)$ & $0(0)$ & $>0.05$ \\
\hline Elevated C-reactive protein $(>8 \mathrm{mg} / \mathrm{dL})$ & $1(5)$ & $1(20)$ & $0(0)$ & $>0.05$ \\
\hline Elevated IL-6 (> 20.9 mg/dL) & $1(5)$ & $1(20)$ & $0(0)$ & $>0.05$ \\
\hline Elevated PCT (>0.1 mg/dL) & $3(15)$ & $3(60)$ & $0(0)$ & 0.009 \\
\hline
\end{tabular}

SARS-CoV-2 severe acute respiratory syndrome coronavirus 2, COVID-19 coronavirus disease 2019, NICU neonatal intensive care unit, SGA small for gestational age, $P C I S$ pediatric critical illness score, $C S$ cesarean section, $A F$ amniotic fluid, $I L-6$ interleukin- $6, P C T$ procalcitonin, - none 
Table 2 Standard neurobehavioral tests to confirm the diagnosis of coronavirus disease 2019 infection in newborns

\begin{tabular}{|c|c|c|c|c|c|c|c|c|c|}
\hline Items & Median (range) & Neonate 1 & Neonate 2 & Neonate 3 & Neonate 4 & Neonate 5 & Control & $Z$ score & $P$ \\
\hline Posture and tone & $40(35-48)$ & 40 & 42 & 38 & 35 & 48 & $42(38-50)$ & 1.793 & 0.0729 \\
\hline Tone patterns & $21(16-25)$ & 20 & 22 & 21 & 16 & 25 & $23(20-25)$ & 1.288 & 0.1980 \\
\hline Reflexes & $22(20-24)$ & 21 & 23 & 22 & 20 & 24 & $24(22-25)$ & 2.210 & 0.0271 \\
\hline Movements & $13(9-16)$ & 13 & 13 & 11 & 9 & 16 & $14(11-15)$ & 0.977 & 0.3287 \\
\hline Abnormal signs/patterns & $12(9-16)$ & 12 & 13 & 12 & 9 & 16 & $14(12-15)$ & 0.932 & 0.3513 \\
\hline Orientation and behavior & $31(25-32)$ & 31 & 32 & 29 & 25 & 32 & $34(32-35)$ & 2.981 & 0.0029 \\
\hline Total scores & $136(114-162)$ & 136 & 145 & 134 & 114 & 162 & $148(140-158)$ & 1.968 & 0.0491 \\
\hline
\end{tabular}

Table 3 Ages and stages questionnaires screening results in coronavirus disease 2019-infected neonates at 9 months of postnatal age

\begin{tabular}{|c|c|c|c|c|c|c|}
\hline Items & Neonate 1 & Neonate 2 & Neonate 3 & Neonate 4 & Neonate 5 & Shaded zones \\
\hline Communication & 60 & 55 & 55 & 50 & 55 & Outside \\
\hline Gross motor & 55 & 50 & 50 & 45 & 50 & Outside \\
\hline Fine motor & 50 & 45 & 50 & 40 & 50 & Outside \\
\hline Problem solving & 50 & 45 & 45 & 45 & 45 & Outside \\
\hline Personal-social & 50 & 50 & 45 & 40 & 50 & Outside \\
\hline Total scores & 265 & 245 & 245 & 225 & 250 & Outside \\
\hline
\end{tabular}

Table 4 Logistic regression analysis of the main affected brain areas of SARS-CoV-2 infection

\begin{tabular}{lllllll}
\hline Brain region & Symmetry & OR & SE & $Z$ & $P$ & 95\% CI \\
\hline CN $^{\text {a }}$ & Right & -4.43 & 1.18 & -2.83 & 0.009 & -7.91 to -1.37 \\
Thalamus $^{\text {a }}$ & Bilateral & -1.55 & 0.47 & -3.32 & 0.007 & -2.46 to -0.49 \\
PHG $^{\mathrm{a}}$ & Right & -2.07 & 0.83 & -2.28 & 0.031 & -4.03 to -0.27 \\
IFG & Left & 0.74 & 1.41 & 0.42 & 0.610 & -3.05 to 4.28 \\
ACG/SFG & Left & -1.40 & 1.29 & -1.21 & 0.217 & -4.08 to 1.31 \\
MFG & Right & -0.74 & 0.52 & -1.26 & 0.194 & -1.88 to 0.55 \\
MTG & Right & -0.18 & 0.99 & 0.17 & 0.786 & -1.98 to 2.65 \\
ORBinf & Left & 1.11 & 0.79 & 1.09 & 0.207 & -0.81 to 2.79 \\
ORBmed & Left & 2.92 & 1.25 & 1.85 & 0.053 & -0.21 to 6.48 \\
ROL & Left & 1.84 & 1.03 & 1.61 & 0.139 & -0.77 to 4.43 \\
Precuneus (L) & Left & 4.26 & 2.32 & 1.96 & 0.066 & -0.54 to 10.11 \\
Precuneus (R) & Right & 4.25 & 2.24 & 1.75 & 0.079 & -0.82 to 8.82 \\
\hline
\end{tabular}

$S A R S-C o V-2$ severe acute respiratory syndrome coronavirus $2, A C G$ anterior cingulate gyrus, $S F G$ superior frontal gyrus, $C N$ caudate nucleus, IFG inferior frontal gyrus, $M F G$ middle frontal gyrus, $M T G$ middle temporal gyrus, ORBinf orbitofrontal cortex (inferior), ORBmed orbitofrontal cortex (medial), $P H G$ parahippocampal gyrus, $R O L$ left Rolandic operculum, $S E$ standard error, $C I$ confidence interval, $O R$ odds ratio, ${ }^{\text {a }}$ The brain area significantly affected by the infection $(P<0.05)$

\section{Which are the major impact brain regions}

The logistic regression model showed (Table 4) that COVID19 infection may affect three brain regions in neonates: thalamus, PHG and CN, showing a decrease in GM volumes. If COVID-19 infection is already present, it is important to be alert to the possibility that the infection may lead to a reduction in GM volumes in these three brain regions. Table 4 shows that SARS-CoV-2 infection may reduce thalamic volume by 1.55 -fold [ $95 \%$ confidence interval (CI) -2.46 to -0.49 ], $\mathrm{CN}$ volume by 4.43 -fold (95\% CI -7.91 to -1.37 ), and PHG volume by 2.07 -fold (95\% CI -4.03 to -0.27 ). 


\section{Discussion}

The Supplementary Fig. 1 demonstrates the effects of COVID-19 on the human CNS, evidence of which has surfaced over time. From the discovery of SARS-CoV-2 in the patient's CSF in March 2020 to the autopsy finding that the virus was also present in frontal neuronal cells and cerebral vascular endothelial cells, the effects of SARSCoV-2 on the CNS were confirmed. This also suggests the need for further studies on the nervous systems of neonates with COVID-19. To our knowledge, no neuroimaging studies have investigated the effects of SARS-CoV-2 infection on neurons and central structures by quantitative methods of brain region segmentation and brain volume calculation. This study is the first to compare the neurological performance of neonates with and without COVID-19 by quantitative neuroimaging and neurological examination methods.

Four of the five infected neonates had abnormalities in cranial MRI. The fourth case was a 31-week gestational age premature infant, and two of the remaining three cases had hypoxic changes; the fifth case had abnormal signals in the bilateral ventricular WM region. The four neonates with abnormal MR signals did not show any obvious signs of hypoxia or asphyxia after birth. It should be further explored whether the hypoxic changes shown in MRI are already present in utero.

In the comparison of brain volumes between the two groups, we found 12 structural areas in which brain volumes differed. Six of these structures had increased brain volumes compared to the control group, and six structures had decreased brain volumes compared to the control group. The changes in the thalamus and precuneus were symmetrical, and the changes in nine brain regions [PHG, ACG, CN, inferior frontal gyrus (IFG), MFG, MTG, inferior ORB, medical ORB, and ROL] were unilateral. Since the two groups also differed in HNNE scores $(P<0.05)$, we analyzed the correlations between brain regions with differences and HNNE scores. We correlated the brain regions with differences in HNNE scores and found that the $\mathrm{CN}, \mathrm{PHG}$, and thalamus had the best positive correlations with HNNE scores. This means that the larger the brain volume of the $\mathrm{CN}$, PHG and thalamus, the higher the HNNE score. This suggests that SARS-CoV-2 may affect neurobehavioral changes in neonates by affecting these sites.

The thalamus plays an important role in human physiology, with each of its nuclei having unique functions, from relaying sensory and motor signals to regulating consciousness and alertness [14]. The site is also prone to brain injury when hypoxia or infection occurs. Recent authors have summarized the neurological manifestations in 78 of 214 (36.4\%) patients, including symptoms of headache, dizziness, impaired consciousness, ataxia, acute cerebrovascular disease, and epilepsy [5]. In another study, this proportion was even higher (77/125) [4]. Therefore, we believe that the thalamus may be a neurological site of potential concern for patients with COVID-19.

However, four of the five SARS-CoV-2-infected neonates (except for one case of 31-week prematurity) had no history of intrauterine distress, asphyxia, or resuscitation after birth; one infected preterm infant received oxygen therapy at admission, but there was no statistical difference between the two groups on oxygen therapy factors. None of the five neonates had hyperbilirubinemia, hypoglycemia, electrolyte disturbance, birth injury, or intracranial hemorrhage, so we could exclude other causes of brain changes. However, since the primary target organ for SARS-CoV-2 infection is the lung, it is also important to consider that this alteration may be due to cerebral hypoxia secondary to lung infection. Helms et al. reported 65 patients with SARS-CoV-2 infection treated in an intensive care unit with confusion in $65 \%$, irritability in $69 \%$, corticospinal tract signs in $67 \%$, and sexual dysfunction syndrome in $36 \%$ of patients [2]. Severe intracranial hemorrhages and strokes have also been reported, but unfortunately no viral particles were found in the CSF [15]. A large proliferation of astrocytes was found in the cerebellum and brainstem of SARS-CoV-2-infected patients, indicating that the virus is capable of directly destroying the CNS [8]. Thus, the characteristic changes in the thalamus may be related to infection, but at the same time, the additive effect of secondary hypoxia on the brain due to pulmonary factors remains unknown. If more neonatal cases are identified in the future, the effects of hypoxic factors on the CNS of infected neonates could be further analyzed.

The IFG, MFG, MTG, inferior ORB, and medical ORB are brain regions closely related to taste and smell [16, 17], sensation is projected via primary neurons to the thalamus and then up to these neocortical layers. The inner olfactory region (the olfactory entorhinal cortex) is in the medial part of the hippocampal paraganglia, both parts of the limbic system, and the primary olfactory cortex includes the PHG. According to the mechanism of the olfactory pathway, because the sense of smell passes through many brain regions that process memory functions, whenever a specific smell is recognized, the brain regions that process memory are activated, evoking memories about the smell [18]. During the COVID-19 pandemic, doctors eventually found loss of smell and taste in some infected people who had no other symptoms. A previous study found that the loss of smell caused by COVID-19 may be related to relatively high expression of angiotensin-converting enzyme 2 receptors in olfactory nerve epithelial cells, which may 
be a potential entry point for the virus to enter the CNS and cause neurological symptoms [19]. A large, multicenter European study found that up to $85 \%$ of patients with COVID-19 had olfactory impairment [20].

Several experiments in transgenic mice have demonstrated that the SARS [21, 22] and Middle East respiratory syndrome [23] viruses are able to penetrate the human brain and to infect the olfactory nerve, which can quickly spread to the thalamus and brainstem. More notably, in mice infected with SARS-CoV-1 (responsible for classic SARS), the virus was found only in the brain and not in the lungs, suggesting that coronaviruses may have a special affinity for the CNS. A research team in China reviewed previous studies and hypothesized that SARS-CoV-2 has the ability to infect the CNS, particularly the nerve regions that process smell and taste [24]. Therefore, the differential changes in the PHG, IFG, MFG, MTG, inferior ORB, and medial ORB shown in Supplementary Fig. 3 may not be coincidental, and the effect of COVID-19 on these brain regions and their prognosis deserve further study.

Notably, we do not advocate the use of differential alterations in brain volume to predict SARS-CoV-2 infection, and in fact the diagnosis of SARS-CoV-2 infection requires definitive laboratory nucleic acid results as clinical evidence. We cannot presume to predict viral infection by volume changes in some characteristic brain regions, which is not in line with clinical practice. In fact, not only infection but also many factors may cause neurological and brain volume alterations. This study only collected data from five neonates with COVID-19 and was limited by the sample size. The results obtained in this study need to be further validated by larger sample sizes or by basic neuroscience studies. Moreover, we did not perform follow-up neuroimaging and neurological examinations of the neonates. The results at one time point do not mean that it will have an impact on the prognosis of the neonates, and we will continue to monitor the neurobehavioral development of these neonates in subsequent follow-up. We would like to conduct more similar studies in different age groups to focus on the structural or functional aspects of SARS$\mathrm{CoV}-2$ in the CNS in the future.

Supplementary Information The online version contains supplementary material available at https://doi.org/10.1007/s12519-021-00423-2.

Author contributions YK, XFF, and JYW contributed equally to this work. YK, XFF, SJB, CGQ, and ZLK conceptualized the study and analyzed the data. JYW, XTT, ZDJ, and YWH co-conceptualized the study and interpreted the data. All authors drafting the article and revising it critically for important intellectual content. All authors revised and approved the final manuscript.

Funding None.

\section{Compliance with the ethical standards}

Ethical approval The study was approved by the local institutional ethics committee at each participating hospital and informed written consent was obtained from the guardians of all participating neonates before the study began.

Conflict of interest The authors have no conflict of interest to declare. No financial or nonfinancial benefits have been received or will be received from any party related directly or indirectly to the subject of this article.

\section{References}

1. Chen N, Zhou M, Dong X, Qu J, Gong F, Han Y, et al. Epidemiological and clinical characteristics of 99 cases of 2019 novel coronavirus pneumonia in Wuhan, China: a descriptive study. Lancet. 2020;395:507-13.

2. Helms J, Kremer S, Merdji H, Clere-Jehl R, Schenck M, Kummerlen C, et al. Neurologic features in severe SARS-CoV-2 infection. N Engl J Med. 2020;382:2268-70.

3. Huang C, Wang Y, Li X, Ren L, Zhao J, Hu Y, et al. Clinical features of patients infected with 2019 novel coronavirus in Wuhan, China. Lancet. 2020;395:497-506.

4. Varatharaj A, Thomas N, Ellul MA, Davies NWS, Pollak TA, Tenorio EL, et al. Neurological and neuropsychiatric complications of COVID-19 in 153 patients: a UK-wide surveillance study. Lancet Psychiatry. 2020;7:875-82.

5. Mao L, Jin H, Wang M, Hu Y, Chen S, He Q, et al. Neurologic manifestations of hospitalized patients with coronavirus disease 2019 in Wuhan, China. JAMA Neurol. 2020;77:683-90.

6. Virhammar J, Kumlien E, Fällmar D, Frithiof R, Jackmann S, Sköld MK, et al. Acute necrotizing encephalopathy with SARSCoV-2 RNA confirmed in cerebrospinal fluid. Neurology. 2020;95:445-9.

7. Paniz-Mondolfi A, Bryce C, Grimes Z, Gordon RE, Reidy J, Lednicky J, et al. Central nervous system involvement by severe acute respiratory syndrome coronavirus-2 (SARS-CoV-2). J Med Virol. 2020;92:699-702.

8. Matschke J, Lütgehetmann M, Hagel C, Sperhake JP, Schröder AS, Edler C, et al. Neuropathology of patients with COVID19 in Germany: a post-mortem case series. Lancet Neurol. 2020;19:919-29.

9. Vivanti AJ, Vauloup-Fellous C, Prevot S, Zupan V, Suffee C, Do $\mathrm{Cao}$ J, et al. Transplacental transmission of SARS-CoV-2 infection. Nat Commun. 2020;11:3572.

10. Raschetti R, Vivanti AJ, Vauloup-Fellous C, Loi B, Benachi A, De Luca D. Synthesis and systematic review of reported neonatal SARS-CoV-2 infections. Nat Commun. 2020;11:5164.

11. Zeng L, Xia S, Yuan W, Yan K, Xiao F, Shao J, et al. Neonatal early-onset infection with SARS-CoV-2 in 33 neonates born to mothers with COVID-19 in Wuhan, China. JAMA Pediatr. 2020;174:722-5.

12. Xiao F, Chen B, Xiao T, Lee SK, Yan K, Hu L. Children with SARS-CoV-2 infection during an epidemic in China (outside of Hubei province). Ann Transl Med. 2020;8:849.

13. Hornman J, Kerstjens JM, de Winter AF, Bos AF, Reijneveld SA. Validity and internal consistency of the Ages and Stages Questionnaire 60-month version and the effect of three scoring methods. Early Hum Dev. 2013;89:1011-5. 
14. Torrico TJ, Munakomi S. Neuroanatomy, thalamus. Florida: StatPearls Publishing LLC; 2020.

15. Al Saiegh F, Ghosh R, Leibold A, Avery MB, Schmidt RF, Theofanis T, et al. Status of SARS-CoV-2 in cerebrospinal fluid of patients with COVID-19 and stroke. J Neurol Neurosurg Psychiatry. 2020;91:846-8.

16. Obiefuna S, Donohoe C. Neuroanatomy, nucleus gustatory. Florida: StatPearls Publishing LLC; 2020.

17. Zou LQ, van Hartevelt TJ, Kringelbach ML, Cheung EFC, Chan RCK. The neural mechanism of hedonic processing and judgment of pleasant odors: an activation likelihood estimation metaanalysis. Neuropsychology. 2016;30:970-9.

18. Helwany M, Bordoni B. Neuroanatomy, cranial nerve 1 (olfactory). Florida: StatPearls Publishing LLC; 2020.

19. Chen M, Shen W, Rowan NR, Kulaga H, Hillel A, Ramanathan $\mathrm{M} \mathrm{Jr}$, et al. Elevated ACE-2 expression in the olfactory neuroepithelium: implications for anosmia and upper respiratory SARS-CoV-2 entry and replication. Eur Respir J. 2020;56:2001948.

20. Parma V, Ohla K, Veldhuizen MG, Niv MY, Kelly CE, Bakke AJ, et al. More than smell-COVID-19 is associated with severe impairment of smell, taste, and chemesthesis. Chem Senses. 2020;45:609-22.

21. Netland J, Meyerholz DK, Moore S, Cassell M, Perlman S. Severe acute respiratory syndrome coronavirus infection causes neuronal death in the absence of encephalitis in mice transgenic for human ACE2. J Virol. 2008;82:7264-75.

22. McCray PB Jr, Pewe L, Wohlford-Lenane C, Hickey M, Manzel L, Shi L, et al. Lethal infection of K18-hACE2 mice infected with severe acute respiratory syndrome coronavirus. J Virol. 2007;81:813-21.

23. Li K, Wohlford-Lenane C, Perlman S, Zhao J, Jewell AK, Reznikov LR, et al. Middle east respiratory syndrome coronavirus causes multiple organ damage and lethal disease in mice transgenic for human dipeptidyl peptidase 4. J Infect Dis. 2016;213:712-22.

24. Li YC, Bai WZ, Hashikawa T. The neuroinvasive potential of SARS-CoV2 may play a role in the respiratory failure of COVID19 patients. J Med Virol. 2020;92:552-5.

Publisher's Note Springer Nature remains neutral with regard to jurisdictional claims in published maps and institutional affiliations. 